\title{
ПРОБЛЕМЫ ДИСТАНЦИОННОГО ОБУЧЕНИЯ И МЕТОДЫ ИХ РЕШЕНИЯ
}

\author{
Макогонюк P.B. ${ }^{1}$ \\ ${ }^{1}$ Одеська Національна академія харчових технологій, Одеса.
}

Copyright (C 2014 by author and the journal “Automation technological and business - processes”. This work is licensed under the Creative Commons Attribution International License (CC BY). http://creativecommons.org/licenses/by/4.0/

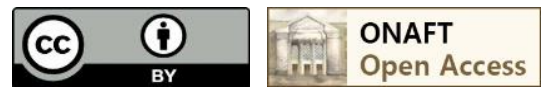

\section{Аннотация \\ В статье рассматриваются проблемы дистанционного обучения и методы их решения.}

\begin{abstract}
The problems of distance learning methods and their solutions.
\end{abstract}

Ключевые слова

Дистанционное обучение, решение, скрипт.

\footnotetext{
Описание

Дистанционное обучение перспективно. Оно постоянно совершенствуется, но существует ряд проблем, исследование которых ведется в настоящее время. Появляются новые методы и пути их решения.

Рассмотрим основные проблемы, связанные в той или иной мере с использованием программ дистанционного обучения. Практически во всех перечисленных программах эти проблемы или не разрешены, или решены частично. В основе технологии дистанционного обучения лежит целенаправленная подготовка студентов к сдаче экзаменов по той или иной теме с использованием различных средств, таких как видео и аудио кассеты, мультимедийные курсы на $\mathrm{CD}$, электронные учебники, электронное тестирование.

Обучение происходит с помощью трех технологий - видео-технологии, кейс-технологии и интернеттехнологии (сетевой технологии). Исследовав основные особенности, преимущества и недостатки различных технологий и программ дистанционного обучения, можно сформулировать ряд основных проблем, характерных для систем дистанционного обучения и дистанционного контроля.

Эти или подобные проблемы возникают у всех, кто занимается созданием систем дистанционного обучения. Большинство из них связано не с разработкой самих учебных материалов для студентов, а с образом контроля полученных знаний. Поэтому больше внимания в работе уделено тому, как преподнести учебную информацию студенту и как проконтролировать его самостоятельную работу. Форма преподнесения информации должна быть удобной, доступной и соответствовать основным принципам дистанционного обучения, а система контроля должна быть эффективной.

Основное проблемы

Сформулируем основные проблемы, связанные с защитой, и ряд других вопросов, относящихся к системам дистанционного обучения.

1. Отсутствие возможности достоверно определить, прошел студент тестирование самостоятельно. Для этой задачи он вполне мог использовать другого человека (например, более подготовленного студента).

2. Неизвестно, сколько раз студент предпринял попытку пройти тестирование. Студент имеет возможность устанавливать систему дистанционного обучения в нескольких экземплярах и / или копировать ее, тем самым сохраняя ее текущее состояние. Так студент получает возможность
} 


\section{7 СТУДЕНТСЬКА НАУКА}

неограниченного количества попыток прохождения тестирования и возможность выбрать из них попытку с наилучшим результатом. Проблема характерна для кейс-технологии и практически отсутствует в интернет-технологии.

3. Очень важной проблемой в области организации самостоятельной работы и, особенно, компьютерного внешнего контроля является слабая защищенность образовательного программного обеспечения от "взлома" с целью доступа к правильным ответам и подделки результатов контроля. Существует возможность создания универсального редактора файлов результатов тестирования. Он может использоваться студентом для корректировки оценок выставленных программой тестирования. Эта проблема вытекает из того, что в основном современные контролирующие системы используют память компьютера для хранения эталонных ответов вместе с заданиями. Как правило, они шифруются, но, как показывает практика, их всегда можно расшифровать. Эта проблема особенно остро встала с появлением в России дистанционных технологий обучения, где внешний контроль знаний осуществляется в основном компьютером в отсутствие преподавателя.

4. Существует угроза создания универсальной программы просмотра файлов с заданиями и ответами. Тем самым, студент имеет возможность узнать верные ответы на вопросы в тестах.

5. Существует также проблема защиты обучающего программного обеспечения от модификации его кода, с целью изменения алгоритма оценивания результатов тестирования или другого кода. Слабая защищенность от "взлома" любых контролирующих систем создает трудности при проведении контроля в системах дистанционного образования. Внешний контроль на расстоянии исключен, потому что никто не может гарантировать, что контролирующие программы не были "сломаны" в процессе выполнения контрольной работы.

Обзор методов решения проблем дистанционного обучения и обоснование выбора решения

Система дистанционного образования должна иметь достаточные средства защиты от несанкционированного вмешательства и подмены реальных студентов их дублерами. Практически отсутствует возможность определить, прошел студент тестирование самостоятельно. Для этой задачи он вполне мог использовать другого человека (например, более подготовленного студента).

При использовании данной системы в аудиториях кафедры, никаких сложностей не возникает, так как студенты находятся под контролем преподавательского состава. Но ориентация образования на дистанционное обучение вносит свои коррективы. Возникает потребность в возможности использования данного программного обеспечения студентом на своей локальной машине. Это, пожалуй, самая сложная задача. Невозможно помешать студенту пойти к другу и попросить выполнить определенную лабораторную работу, пройти тестирование. Без применения специальной аппаратуры это практически нереально. Но, естественно, применение аппаратных средств невозможно в силу хотя бы своей высокой цены. Следовательно, такой вариант рассматриваться не будет. По крайней мере, он не реальный да данном этапе состояния образования в нашей стране.

Студент может посадить за компьютер вместо себя более осведомленного в предмете человека. Навигационная система ГО должна проверять, находится за удаленным компьютером именно обучаемый, за которого он себя выдает, то есть, сделать распознавания пользователя.

Каким образом сегодня решается эта проблема? Каждый поступающий на обучение в СДО получает свое входное имя и пароль для входа на сервер с учебными материалами. При обращении ученика к серверу о нем можно собирать информацию, полезную для преподавателя: перечень страниц, посещенных пользователем за сеанс работы; время, проведенное на каждой странице; активированные гиперссылки на данной странице; перечень файлов, которые были скопированы пользователем с учебного сервера; время тестирования и др..

При необходимости администратор сервера СДО может с помощью собираемой восстановить любой сценарий сеанса работы какого-либо обучаемого.

Но вся собранная таким образом информация является косвенной. То есть, если в систему вошел человек по входному имени и паролю своего коллеги с целью отметиться и принять участие в тестировании, то его невозможно разоблачить. Иными словами, нужны прямые доказательства того, что данный сеанс обучения провел действительно тот пользователь, с чьим именем сопоставлены входное имя и пароль.

Другой вариант, в общении с компьютером индивидуальность пользователя проявляется в скорости, привычкой использовать основную или дополнительную часть клавиатуры, характере "сдвоенных" и "строенных" нажатий клавиш, любимых приемов работы с компьютером, с помощью которых можно выделить конкретного человека среди работали на данной машине. Разумеется, по двум-трем, даже по десяти нажатым клавишам отличить пользователя невозможно, нужна статистика. 
При наборе ключевой фразы компьютер позволяет зафиксировать много различных параметров, но для идентификации наиболее удобно использовать время, затраченное на ввод отдельных букв. А повторив ввод фразы несколько раз, в результате получим множество временных интервалов для каждого символа. На базе полученных значений всегда можно рассчитать среднее время ввода каждого символа, допустимое отклонение от среднего, и хранить эти результате как эталоны для каждого пользователя. Это требует разработки сложных алгоритмов проверки и идентификации и значительных материальных затрат. К тому же это актуально при достаточно длительной работе пользователя на компьютере. При студента этого практически нет, при своей работе ему придется вводить совсем немного текстовых данных. Подобная идентификация студента для данной работы также не приемлема.

Можно осуществлять тестирование в подготовленной аудитории под контролем преподавателя, в условленное время, но это затрагивает основной принцип дистанционного обучения - возможность получения знаний и их контроля в удобном месте и в удобное время для каждого студента. Хотя этот вариант исключать нельзя, так как студент изучает материалы курса дистанционно.

На основании вышесказанного, кажется, что проблема идентификации студентов, вообще не имеет решения. Действительно, как уже отмечалось, пароли и прочие атрибуты личной идентификации проблемы не решают, т.к. студент заинтересован заменить себя дублером и располагает неограниченными возможностями такой подмены. Дешевое техническое решение для ее обнаружения придумать пока не удалось.

Выход может быть в создании постепенного поэтапного контроля знаний с целью формирования трудностей для подмены проходит тестирование дублером. Следующий этап обучения и доступ к последующим материалами курса, будет доступен только при успешной сдаче контрольного теста предыдущего. Найти дублера на один экзамен намного проще, чем на весь период обучения. Такая задача может быть решена (и решается достаточно хорошо) с применением сетевых технологий. В такой системе студент заходит на сайт, изучает определенный раздел курса, проходит тестирование по этому разделу и только в случае успешной сдачи теста получает возможность двигаться дальше.

\section{Выводы}

1. В любом случае должен присутствовать очный итоговый контроль, так как тестирование это лишь промежуточный этап, прохождение которого дает студенту допуск к зачету или экзамену. Итоговый контроль курса должен осуществляться при личном контакте преподавателя и студента;

2. Неизвестно, сколько раз студент предпринял попытку пройти тестирование. Студент имеет возможность устанавливать систему дистанционного обучения в нескольких экземплярах и / или копировать ее, тем самым сохраняя ее текущее состояние. Студент получает возможность неограниченного количества попыток прохождения тестирования и выбора из них попытки с наилучшим результатом. Необходимо применение различных ухищрений при хранении информации о проделанной студентом работе. Решить эту проблему не просто. В любом случае невозможно узнать, что студент установил пакет программ дистанционного обучения на двух компьютерах, а затем использует один для тренировки и подбора правильных ответов, а второй уже для тестирования. Причем он может поступить проще и воспользоваться программой для создания множества виртуальных машин на одной физической. Такой программой, например, является WMware. Или может создать одну виртуальную машину и установить на ней программу, которая производит тестирование. А потом, если результат прохождения тестирования его не будет устраивать, он сможет восстановить предыдущее состояние всей виртуальной системы, просто сделав откат. Одним из методов решения данной проблемы может стать использование индивидуальной дискеты студента. Идея здесь та же, что и использовалась при защите программного обеспечения от несанкционированного копирования. Сейчас для этих целей все чаще используется технология электронных ключей (Hasp и т.д.). Смысл в том, что дискета форматируется, а затем используется особым образом. То есть работать с такой дискетой может только специальный набор программ. Стандартными методами такую дискету нельзя ни скопировать, ни просмотреть информацию на ней, так как она хранится в особом формате. Работать с такой дискетой могут только программы, входящие в состав системы дистанционного обучения. Без ключевой дискеты система работать не будет. На этой дискете можно отмечать количество попыток тестирования т.д., тем самым, решая проблему, которая рассматривается. Но эта технология не является решением проблемы. Дискета может быть скопирована программой, например, COPYWRIT, и т.п. Достаточно легко воспользоваться специализированными программами копирования или создать свой аналог. Отсюда можно сделать вывод, что разработка системы с использованием ключевой дискеты нерациональна. Подобный подход также повлечет массу 
сложностей. Например, не ясно, что делать в случае утери или порчи дискеты. Но, как видно, в этом случае студенту придется проходить тестирование еще раз, что явно неприемлемо. Можно сделать вывод, что без использования специальных аппаратных средств, данная проблема также не имеет достаточно удовлетворительного решения. Но к ее решению можно постараться приблизиться, используя специфические методы хранения состояния (например, в реестре и т.п.), тем самым усложняя процесс махинации. Но в общем случае это опять не даст результата, если студент воспользуется виртуальной машиной, созданной, например, программой WMware. Решением проблемы может быть хранение проб и результатов тестирования в базе данных, расположенной на web-сервере, доступ к изменению значений которой может получить только администратор системы. Это наиболее оптимальный и безопасный вариант;

3. Существует возможность создания универсального редактора файлов результатов тестирования. Он может использоваться студентом для корректировки оценок выставленных программой тестирования. Здесь можно было воспользоваться идеей ключевой дискеты для сохранения результата. Тем самым модификация результата стала бы очень трудной. Но этот метод имеет одно ограничение, которое делает его практически непригодным. Это необходимость использования для передачи результата дискеты. То есть вместо того, чтобы просто отправить результат по сети, придется доставлять его на дискете. Но есть другой метод. Это использование шифрования. Как бы ни были сложны и надежны криптографические системы - их слабое место при практической реализации - проблема распределения ключей. Для того, чтобы был возможен обмен конфиденциальной информацией между двумя субъектами ИС, ключ должен быть сгенерирован одним из них, а затем каким-то образом опять же в конфиденциальном порядке передан другому. Т.е. в общем случае для передачи ключа опять же требуется использование какой-то криптосистемы. Еще один вариант решения поставленной задачи использование сети Интернет и web-интерфейса. Результаты сразу же передаются на web-cepвер, и обрабатываются уже непосредственно там, тем самым исключается возможность редактирования результата, так как доступ к базе данных результатов тестирования имеет только администратор системы;

4. Существует возможность создания универсальной программы просмотра файлов с заданиями и ответами. Таким образом, студент имеет возможность узнать верные ответы на вопросы в тестах. Естественным выходом из данной ситуации является применение шифрования данных. Но принципиально данную проблему решить невозможно. Студенту необходимо поставить вопрос и сверить с ответом, а для этого необходимо расшифровать данные с эталонными ответами. Для их расшифровки необходим ключ, который в любом случае надо где-то хранить. Следовательно, при желании, информацию можно получить в открытом виде. Побочного проблемой является возможность внесения заинтересованным лицом несанкционированного изменения баз данных обучающих систем. Решить эту проблему частично можно, разграничив клиентскую и администраторскую части программного комплекса. В клиентской части нет возможности доступа к базе данных для ее изменения - это возможно с администраторской части при знании пароля доступа к базе данных. Для работы клиентской части необходима заполненная база данных тестовыми заданиями. Это должно быть сознательно сделано из части администратора. Таким образом, существует защита от модификации, но нет защиты от просмотра. Так же, совершенно очевидно, отсутствует и защита отчетов о результатах тестирования. Эту проблему можно решить, воспользовавшись преимуществами сетевой технологии, например, разместив базу данных вопросов и ответов на web-cepвере, а доступ к ней организовать средствами языка web-программирования, формирующего готовые страницы данных, запрашиваемые пользователем на стороне сервера. При этом посмотреть данные сможет только администратор системы, а пользователь получит только ту ограниченную их часть, которая предназначена именно для него;

5. Возможность модификации программного кода системы тестирования с целью изменения алгоритма выставления оценок. Как ни странно, но столь важный вопрос защиты практически не рассматривается. Отчасти это понятно. Для систем построенных с использованием сети Интернет, его практически не существует. Так как контролирующая часть находится на стороне сервера, то данная проблема не актуальна. Для систем дистанционного обеспечения, предназначенных для локального режима использования, как это реализовано в кейс технологии, эта проблема практически сводится к широко известной проблемы защиты ПО от взлома. 
Это еще один плюс использования сетевой технологии для решения задач, поставленных в этой работе. Таким образом хорошим решением для многих из перечисленных проблем являются:

1. Использование интернет-технологии и создание web-приложения;

2. Поэтапное преподнесения информации и поэтапный контроль знаний;

3. Глазной итоговый контроль знаний;

4. Хранение вопросов и ответов, проб и результатов тестирования в базе данных, расположенной на wеbсервере, доступ к просмотру и редактированию которой есть только у определенного круга лиц;

5. Разграничение администраторской и клиентской частей приложения;

6. Использование для разработки системы скриптовых языков программирования, формируют страницу с данными непосредственно на web-сервере. Пользователь системы получает страницу, содержащую текст подготовлен лично для него.

Научный руководитель: стариий преподаватель Дец Д.В.

\title{
Литература
}

1. Адлер Ю.П., Кочетов А.И. Повышение качества подготовки специалистов.// Стандарты и качество, МИСиС - 2000.- № 2.- С. 68-72;

2. Куклев, В. А. Методология мобильного обучения. - Ульяновск: УлГТУ, 2006. - 254 с.

\section{ИСПОЛЬЗОВАНИЕ ШАБЛОНА ПРОЕКТИРОВАНИЯ MVC ДЛЯ СОЗДАНИЯ ВЕБ-ПРИЛОЖЕНИЙ}

\author{
Щербакан Д.В. ${ }^{1}$ \\ ${ }^{1}$ Одеська Національна академія харчових технологій, Одеса.
}

Copyright (C) 2014 by author and the journal “Automation technological and business - processes". This work is licensed under the Creative Commons Attribution International License (CC BY). http://creativecommons.org/licenses/by/4.0/

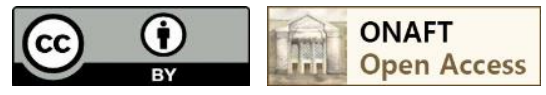

\section{Аннотация \\ Ррассматриваются общие принципы построения приложений с использованием шаблона проектирования MVC. Указываются некоторые особенности его использования в веб-разработке.}

\begin{abstract}
The general principles for building applications using the MVC design pattern are reviewing. Some features of using it in web-development are specifying.
\end{abstract}

Ключевые слова

Программирование, MVC, веб-разработка.

Постановка проблемы.

На данном этапе развития интернета, все чаще веб-приложения должны реализовывать сложную бизнеслогику, передавать и обрабатывать логически связанные данные, автоматически реагировать на возникновение ошибок. При этом необходимо сохранить возможность быстрого изменения или добавления функционала 